\title{
Cryptanalysis and security enhancement on the generation of Mu-Varadharajan electronic voting protocol
}

\section{Vahid Jahandideh and Amir S. Mortazavi}

Department of Electrical Engineering, Sharif University of Technology, Tehran, Iran

E-mail: v_jahandideh@ee.sharif.edu

E-mail: sa_mortazavi@ee.sharif.edu

\section{Yaser Baseri* and Javad Mohajeri}

Electronics Research Center, Sharif University of Technology,

Tehran, Iran

Fax: +98-21-66030318

E-mail: yaser_baseri@alum.sharif.ir

E-mail: mohajer@sharif.edu

${ }^{*}$ Corresponding author

\begin{abstract}
Mu}$ and Varadharajan proposed an electronic voting scheme and claimed that their scheme authenticates the Voters, protects the anonymity of them, and detects the identity of double voters. Due to some weaknesses in Mu-Varadharajan scheme, several modified schemes have been proposed by Lin et al., Hwang et al., Rodríguez-Henríquez et al. and Asaar et al.; however this paper shows that these schemes suffer from some weaknesses in fulfilling the pointed properties. For this purpose, we get Hwang et al. scheme as a case study and apply our new attacks on it. Also we consider the applicability of the attacks on other pointed schemes. In addition, we present a new scheme and show that the scheme resists against the proposed attacks without loosing efficiency.
\end{abstract}

Keywords: electronic voting; cryptanalysis; anonymity; unforgeability; perceptibility of double voting; attack prevention.

Reference to this paper should be made as follows: Jahandideh, V., Mortazavi, A.S., Baseri, Y. and Mohajeri, J. (2010) 'Cryptanalysis and security enhancement on the generation of Mu-Varadharajan electronic voting protocol', Int. J. Electronic Governance, Vol. 3, No. 1, pp.72-84.

Biographical notes: Vahid Jahandideh received his BS Degree in Electrical Engineering in 2008 from Tabriz University. He is currently pursuing his MS Degree in Department of Electrical Engineering of Sharif University of Technology. His research interest includes cryptography, information security, and information theory.

Copyright (c) 2010 Inderscience Enterprises Ltd. 
Amir S. Mortazavi received his BS Degree in Electrical Engineering in 2008 from Tabriz University. He is currently continuing his master level in communication cryptography field in Sharif University of Technology. His major research interests include information theory, cryptography, and speech enhancement.

Yaser Baseri received his BSc Degree in Computer Science from Shahid Beheshty University, Tehran, Iran, in 2005 and the MSc Degree in Computer Science from Sharif University of Technology, Tehran, Iran, in 2007. He is currently doing research in Electronics Research Center, Sharif University of Technology. His research interests are formal method, cryptography and network security.

Javad Mohajeri received the BSc Degree from the Isfahan University in 1986 and MSc Degree from Sharif University of Technology in 1989, both in Mathematics. Since 1990, he has been a Faculty Member at Electronics Research Center of Sharif University of Technology. His research interests include cryptography and data security. $\mathrm{He}$ is author/co-author of over 45 research articles in refereed Journals/Conferences. He is one of the Founding Members of Iranian Society of Cryptology.

\section{Introduction}

By the need of democracy in developing human societies and by developing communication networks and internet, it is necessary to produce new secure e-voting schemes that satisfy some requirements such as anonymity of voters, unforgeability of tickets and perceptibility of double voters.

Electronic voting protocols could be classified by their approach into three categories:

1 blind signature based electronic voting schemes (Chum, 1988; Fujioka et al., 1988; Juang and Lei, 1997; Lee et al., 2004)

2 homomorphic encryption based electronic voting schemes (Benaloh, 1987; Hirt and Sako, 2000)

3 electronic voting schemes which use randomisation such as the schemes which employ mixnets (Chaum, 2004; Chaum et al., 2005).

One of the first voting protocols which is based on blind signature and used to claim that it could protect voter's anonymity, authenticate the voters and detect the identity of double voters, is proposed by $\mathrm{Mu}$ and Varadharajan (1998). They also claimed that their scheme is suitable for large-scale elections. In Chien et al. (2003) showed that there are some weaknesses in security of Mu-Varadharajan's schemes such as identifying the owner of a cast ballot by authorities, imperceptibility of double voting and forgeability of a ballot by anyone without being authenticated. In Lin et al. (2003) proposed an improvement on Mu and Varadharajan's protocol. They have tried to improve the weakness that voters could successfully vote more than once without being detected. Their proposed scheme does not require any 
special voting channel and it is claimed that the scheme is able to detect vote duplicity effectively. Yang et al. (2004) proposed another improvement on $\mathrm{Mu}$ and Varadharajan's protocol. Although their scheme is resistant to the attacks which has been proposed in Chien et al. (2003), it can not determine the identity of double voter. Hwang et al. (2005) represented an attack on Lin et al. protocol in Hwang et al. (2005). They also showed that the modification of Lin et al. allows the Authentication Server (AS) to identify the voters of published tickets so that voters will lose their privacy. Hwang proposed a new scheme to solve this problem and thus enhance the security (Hwang et al., 2005). It was claimed that the protocol satisfies the privacy of voters and detects double voting. In Asaar et al. (2008a) showed that the Hwang et al. scheme has the weakness that eligible voter with a valid ticket could vote more than once without being detected. They also proposed a new improvement on it. However it is possible to show that their improvement has the same weaknesses as the weaknesses which are proposed in this paper. Furthermore in Rodríguez-Henríquez et al. (2007) proposed another improvement over the $\mathrm{Mu}$ and Varadharajan e-voting protocol which suffers from the same problem as Hwang et al. scheme. Moreover, in Asaar et al. (2008b) proposed another scheme based on Lin et al. scheme. Although, their scheme resist against the proposed attacks in Chien et al. (2003), it is vulnerable to some attacks which are presented in Section 2.2.

In this paper first we review Hwang et al. scheme and its failures in Section 2. Then in Section 3 we propose a new modified scheme to solve the weaknesses of the former schemes and show that the new scheme would not be affected by the proposed attack without losing efficiency and requirement of the former one. The conclusion is presented in Section 4.

\section{Hwang et al. scheme and its failures}

In this section first we describe the protocol proposed by Hwang et al. in Subsection 2.1. Then in Subsection 2.2 we present some attacks on anonymity of voter, unforgeability of ticket and perceptibility of double vote in this scheme. The applicability of these attacks to some other schemes on the race of $\mathrm{Mu}$-Varadharajan scheme are presented.

\subsection{Hwang et al. scheme}

The Hwang et al.'s anonymous electronic voting scheme consists of the following participants: Voters $(V)$, an Authentication Server $(A S)$, Voting Servers $(V S)$, a Ticket Counting Server $(T C S)$, and a Certificate Authority $(C A)$. For convenience, some necessary notation are defined below:

- $\quad\left(e_{x}, n_{x}\right), d_{x}$ : the RSA public/private key pair of participant $x$.

- $C e r t_{x}$ : the public-key certificate of participant $x$, which is signed by $C A$.

- $\quad p$ : a large prime number, which is a public system parameter.

- $\quad g, h$ : are two different elements in $\mathbb{Z}_{p}^{*}$ which are also public system parameters.

- $\|$ : the operation of concatenation.

- $t$ : timestamp. 


\subsubsection{The voting and ticket obtaining phase}

a A voter $V$ chooses two blind factors $b_{1}, b_{2}$ in $\mathbb{Z}_{n_{A S}}^{*}$ and two random numbers $k_{1}$ and $r$ in $\mathbb{Z}_{p}^{*}$. Then, $V$ computes $w_{1}, w_{1}^{\prime}, w_{2}$ and $w_{2}^{\prime}$ as follows:

$$
\begin{aligned}
& w_{1}=g^{r} b_{1}^{e_{A S}} \bmod n_{A S} \\
& w_{1}^{\prime}=h^{r} b_{1}^{e_{A S}} \bmod n_{A S} \\
& w_{2}=g^{k_{1}} b_{2}^{e_{A S}} \bmod n_{A S} \\
& w_{2}^{\prime}=h^{k_{1}} b_{2}^{e_{A S}} \bmod n_{A S} .
\end{aligned}
$$

Then, the voter sends $\left\{V, A S, \operatorname{Cert}_{V}, t, w_{1}, w_{1}^{\prime}, w_{2}, w_{2}^{\prime},\left(\left(w_{1}\left\|w_{1}^{\prime}\right\| w_{2}\left\|w_{2}^{\prime}\right\| t\right)^{d_{V}}\right)\right.$ $\left.\bmod n_{V}\right\}$ to $A S$.

b $A S$ first verifies the validity of the certificate and timestamp, then validate the signature $\left(\left(w_{1}\left\|w_{1}^{\prime}\right\| w_{2}\left\|w_{2}^{\prime}\right\| t\right)^{d_{V}}\right) \bmod n_{V}$. After passing all verifications, $A S$ chooses a unique random number $k_{2}$ and computes:

$$
\begin{aligned}
& w_{3}=\left(k_{2} \| t\right)^{e_{V}} \bmod n_{v} \\
& w_{4}=\left(w_{1} \times A S\right)^{d_{A S}} \bmod n_{A S} \\
& =\left(a_{1} \times A S\right)^{d_{A S}} \times b_{1} \bmod n_{A S} \\
& w_{5}=\left(w_{1}^{\prime} \times A S\right)^{d_{A S}} \bmod n_{A S} \\
& =\left(a_{2} \times A S\right)^{d_{A S}} \times b_{1} \bmod n_{A S} \\
& w_{6}=\left(w_{2} \times g^{k_{2}} \times A S\right)^{d_{A S}} \bmod n_{A S} \\
& =\left(y_{1} \times A S\right)^{d_{A S}} \times b_{2} \bmod n_{A S} \\
& w_{7}=\left(w_{2}^{\prime 2} \times h^{k_{2}} \times A S\right)^{d_{A S}} \bmod n_{A S} \\
& =\left(y_{2} \times A S\right)^{d_{A S}} \times b_{2}^{2} \bmod n_{A S}
\end{aligned}
$$

where $a_{1}=g^{r}, a_{2}=h^{r}, y_{1}=g^{k_{1}+k_{2}}$, and $y_{2}=h^{2 k_{1}+k_{2}}$. Then, the messages $\left\{A S, V, w_{3},\left(w_{4}\left\|w_{5}\right\| w_{6}\left\|w_{7}\right\| t\right)^{e_{V}} \bmod n_{V}\right\}$ are delivered to $V$. Note that $A S$ also stores $k_{2}$ along with $V$ 's identity in its database.

c $\quad V$ obtains $k_{2}$ by decrypting $w_{3}$. Thus, $V$ can calculate $y_{1}$ and $y_{2}$ by using $g, h$, $k_{1}$ and $k_{2}$. Furthermore, $V$ also computes the signatures $s_{1}, s_{2}, s_{3}$ and $s_{4}$ by removing the blinding factors $b_{1}$ and $b_{2}$ from $w_{4}, w_{5}, w_{6}$ and $w_{7}$ as follows:

$$
\begin{aligned}
& s_{1}=w_{4} \times b_{1}^{-1} \bmod n_{A S}=\left(a_{1} \times A S\right)^{d_{A S}} \bmod n_{A S} \\
& s_{2}=w_{5} \times b_{1}^{-1} \bmod n_{A S}=\left(a_{2} \times A S\right)^{d_{A S}} \bmod n_{A S} \\
& s_{3}=w_{6} \times b_{2}^{-1} \bmod n_{A S}=\left(y_{1} \times A S\right)^{d_{A S}} \bmod n_{A S} \\
& s_{4}=w_{7} \times b_{2}^{-2} \bmod n_{A S}=\left(y_{2} \times A S\right)^{d_{A S}} \bmod n_{A S} .
\end{aligned}
$$

d $V$ applies the ElGamal digital signature scheme (ELGamal, 1985) to sign the voting content $m$. Let $x_{1}=k_{1}+k_{2}$ and $x_{2}=2 k_{1}+k_{2}$ be the private keys of ElGamal digital signature and $y_{1}, y_{2}$ be the corresponding public keys such that $y_{1}=g^{k_{1}+k_{2}} \bmod p$ and $y_{2}=h^{2 k_{1}+k_{2}} \bmod p . V$ generates two signature $\left(a_{1}, s_{5}\right)$ and $\left(a_{2}, s_{6}\right)$ of the voting content $m$ by using the following equations:

$s_{5}=x_{1}^{-1}\left(m a_{1}-r\right) \bmod p-1$

$s_{6}=x_{2}^{-1}\left(m a_{2}-r\right) \bmod p-1$. 
Then the voting ticket can be computed as

$T=\left\{s_{1}\left\|s_{2}\right\| s_{3}\left\|s_{4}\right\| s_{5}\left\|s_{6}\right\| a_{1}\left\|a_{2}\right\| y_{1}\left\|y_{2}\right\| m\right\}$

\subsubsection{The voting and tickets collecting phase}

a $\quad V$ sends the voting ticket $T$ to $V S$.

b $V S$ validates $a_{1}, a_{2}, y_{1}$, and $y_{2}$ by checking the following equations:

$$
\begin{aligned}
& A S \times a_{1} \stackrel{?}{=} s_{1}^{e_{A S}} \bmod n_{A S} \\
& A S \times a_{2} \stackrel{?}{=} s_{2}^{e_{A S}} \bmod n_{A S} \\
& A S \times y_{1} \stackrel{?}{=} s_{3}^{e_{A S}} \bmod n_{A S} \\
& A S \times y_{2} \stackrel{?}{=} s_{4}^{e_{A S}} \bmod n_{A S} .
\end{aligned}
$$

If all of the above equations hold, $V S$ further verifies the signatures $\left(a_{1}, y_{1}, s_{5}\right)$ and $\left(a_{2}, y_{2}, s_{6}\right)$ of the voting content $m$ by checking the following equations:

$$
\begin{aligned}
& y_{1}^{s_{5}} a_{1} \stackrel{?}{=} g^{m a_{1}} \bmod p \\
& y_{2}^{s_{6}} a_{2} \stackrel{?}{=} h^{m a_{2}} \bmod p .
\end{aligned}
$$

If both verifications succeed, $V S$ stores $T$ in its database.

c After the voting time expired, $V S$ sends all the collected tickets to TCS.

\subsubsection{The tickets counting phase}

Upon receiving all tickets from the $V S, T C S$ first verifies if there are double voting tickets by checking $y_{1}, y_{2}, a_{1}$ and $a_{2}$ for every ticket to see whether they have been repetitively used. If these parameters appear in more than one ticket, the owner of this ticket has voted twice or more. Moreover, if the voter uses the same parameters to sign different voting contents, $T C S$ and $A S$ can cooperate to find the malicious voter as follows. Assume that $T C S$ discovers a voter using the same parameters $y_{1}, y_{2}, a_{1}$ and $a_{2}$ to sign two different voting contents $m$ and $m^{\prime}$. Then TCS can calculate

$$
\begin{aligned}
& x_{1}=\frac{m^{\prime} a_{1}-m a_{1}}{s_{5}^{\prime}-s_{5}} \bmod (p-1) \\
& x_{2}=\frac{m^{\prime} a_{2}-m a_{2}}{s_{6}^{\prime}-s_{6}} \bmod (p-1) \\
& k_{1}=x_{2}-x_{1}=\left(2 k_{1}+k_{2}\right)-\left(k_{1}+k_{2}\right) \\
& k_{2}=x_{1}-k_{1} .
\end{aligned}
$$

Finally, TCS can identify the malicious voter by searching $A S$ 's database to find out which voter is associated with the unique random number $k_{2}$. Then the TCS publishes the valid tickets and counts them. 


\subsection{Weaknesses of this scheme}

In this subsection we present some attacks on Hwang et al. scheme and show that the scheme is vulnerable to the claimed properties (i.e. anonymity of voter, unforgeability of tickets and perceptibility of double voter). Furthermore applicability of these attacks on other schemes of the family of Mu-Varadharajan scheme are shown in Table 1.

Table 1 Comparing resistance of different schemes on the race of Mu-Varadharajan scheme against the proposed attacks $(\checkmark$ : applicable, $\times$ : unapplicable)

\begin{tabular}{|c|c|c|c|c|c|}
\hline \multirow[b]{2}{*}{ Schemes } & \multicolumn{2}{|c|}{ Attacks on anonymity } & \multicolumn{2}{|c|}{ Attacks on unforgeability } & \multirow{2}{*}{$\begin{array}{c}\begin{array}{c}\text { Attacks on } \\
\text { perceptibility }\end{array} \\
\begin{array}{c}\text { First } \\
\text { attack }\end{array}\end{array}$} \\
\hline & $\begin{array}{c}\text { First } \\
\text { attack }\end{array}$ & $\begin{array}{l}\text { Second } \\
\text { attack }\end{array}$ & $\begin{array}{c}\text { First } \\
\text { attack }\end{array}$ & $\begin{array}{l}\text { Second } \\
\text { attack }\end{array}$ & \\
\hline Lin et al. (2003) & $x$ & $\checkmark$ & $\checkmark$ & $\checkmark$ & $\times$ \\
\hline Asaar et al. (2008b) & $\times$ & $\checkmark$ & $\checkmark$ & $\checkmark$ & $\times$ \\
\hline Hwang et al. (2005) & $\checkmark$ & $\checkmark$ & $\checkmark$ & $\checkmark$ & $\checkmark$ \\
\hline Asaar et al. (2008a) & $\checkmark$ & $\checkmark$ & $\checkmark$ & $\checkmark$ & $\checkmark$ \\
\hline $\begin{array}{l}\text { Rodríguez-Henríquez } \\
\text { et al. (2007) }\end{array}$ & $\times$ & $\checkmark$ & $\times$ & $\checkmark$ & $\times$ \\
\hline
\end{tabular}

\subsubsection{The attacks on anonymity of voter}

First attack. In this scheme since parameters $w_{1}$ and $w_{1}^{\prime}$ are blinded with the same blinding factor, when the voter sends $\left\{V, A S, \operatorname{Cert}_{V}, t, w_{1}, w_{1}^{\prime}, w_{2}, w_{2}^{\prime}\right.$, $\left.\left(\left(w_{1}\left\|w_{1}^{\prime}\right\| w_{2}\left\|w_{2}^{\prime}\right\| t\right)^{d_{V}}\right) \bmod n_{V}\right\}$ to $A S, A S$ can compute the value $z_{1}=\frac{w_{1}}{w_{1}^{\prime}}=\frac{g^{r}}{h^{r}}$ for the voter and store it beside the identity of him. On the other hand at the end of voting process, when TCS publishes tickets $A S$ can compute the value $z_{2}=\frac{a_{1}}{a_{2}}=\frac{g^{r}}{h^{r}}$ for each ticket which is published by TCS. By matching the values of $z_{1}$ and $z_{2}, A S$ can determine the owner of each vote $m$.

Since, Asaar et al. (2008a) has similar structure to this scheme, the attack can also be employed on the scheme. However, since Lin et al. (2003) scheme, Asaar et al. (2008b) scheme and Rodríguez-Henríquez et al. (2007), have used different blinding factors in different equations, computing the value similar to $z_{1}$ is impossible and this attack can not be employed on them.

Second attack. Suppose that TCS has published all cast tickets. In order to find the owner of the ticket $T=\left\{s_{1}\left\|s_{2}\right\| s_{3}\left\|s_{4}\right\| s_{5}\left\|s_{6}\right\| a_{1}\left\|a_{2}\right\| y_{1}\left\|y_{2}\right\| m\right\}$, $A S$ can perform the following procedure to identify the owner of this ticket:

$1 A S$ select a record $\left\{V^{\prime}, k_{2}^{\prime}\right\}$ from its own database and computes the value $r^{\prime}$ as follows:

$$
r^{\prime}=\frac{s_{5} s_{6} k_{2}^{\prime}-m\left(2 a_{1} s_{6}-a_{2} s_{5}\right)}{s_{5}-2 s_{6}} .
$$


If the equation $a_{1} \stackrel{?}{=} g^{r^{\prime}}$ holds then $r^{\prime}=r$ and $V^{\prime}$ is the owner of this ticket; else $A S$ chooses another record from its own data base and compute the equation (8) while the owner of this vote is determined.

2 This step is done while the owner of all tickets are determined.

This attack can be employed on Lin et al. (2003), Hwang et al. (2005), Asaar et al. (2008a, 2008b), and Rodríguez-Henríquez et al. (2007) in similar ways.

\subsubsection{The attacks on unforgeability of tickets}

First attack. Hwang et al. scheme has weakness with respect to a kind of multiplicative attack. Assume that $p>>n_{A S}$. A malicious voter, using a valid ticket, can forge tickets as much as he desires without detection. After ticket obtaining phase, malicious voter choose four numbers $\delta_{1}, \delta_{2}, \delta_{3}, \delta_{4}$ arbitrary and compute the value of $a_{1}^{\prime}, a_{2}^{\prime}, y_{1}^{\prime}, y_{2}^{\prime}, s_{1}^{\prime}, s_{2}^{\prime}, s_{3}^{\prime}, s_{4}^{\prime}$ as forged duplicate values of $a_{1}, a_{2}, y_{1}, y_{2}, s_{1}$, $s_{2}, s_{3}, s_{4}$ by the following procedure:

$$
\begin{aligned}
& a_{1}^{\prime}=a_{1} \times g^{\delta_{1} \cdot e_{A S}} \bmod p \\
& a_{2}^{\prime}=a_{2} \times h^{\delta_{2} \cdot e_{A S}} \bmod p \\
& y_{1}^{\prime}=y_{1} \times g^{\delta_{3} \cdot e_{A S}} \bmod p \\
& y_{2}^{\prime}=y_{2} \times h^{\delta_{4} \cdot e_{A S}} \bmod p \\
& s_{1}^{\prime}=s_{1} \times g^{\delta_{1}} \bmod n_{A S} \\
& s_{2}^{\prime}=s_{2} \times h^{\delta_{2}} \bmod n_{A S} \\
& s_{3}^{\prime}=s_{3} \times g^{\delta_{3}} \bmod n_{A S} \\
& s_{4}^{\prime}=s_{4} \times h^{\delta_{4}} \bmod n_{A S} \\
& x_{1}^{\prime}=x_{1}+\delta_{3} \cdot e_{A S} \\
& x_{2}^{\prime}=x_{2}+\delta_{4} \cdot e_{A S} \\
& s_{5}^{\prime}=x_{1}^{\prime-1} \times\left(m a_{1}^{\prime}-\left(r+\delta_{1} \cdot e_{A S}\right)\right) \bmod (p-1) \\
& s_{6}^{\prime}=x_{2}^{\prime-1} \times\left(m a_{2}^{\prime}-\left(r+\delta_{2} \cdot e_{A S}\right)\right) \bmod (p-1) .
\end{aligned}
$$

Finally the forged duplicate ticket would be $T^{\prime}=\left\{s_{1}^{\prime}\left\|s_{2}^{\prime}\right\| s_{3}^{\prime}\left\|s_{4}^{\prime}\right\| s_{5}^{\prime}\left\|s_{6}^{\prime}\right\| a_{1}^{\prime} \|\right.$ $\left.a_{2}^{\prime}\left\|y_{1}^{\prime}\right\| y_{2}^{\prime} \| m\right\}$. This ticket is passed through all validations which will be done:

$$
\begin{gathered}
\left\{\begin{array}{l}
A S \times a_{1}=s_{1}^{e_{A S}} \bmod n_{A S} \Rightarrow \\
A S \times a_{1}^{\prime}=s_{1}^{\prime e_{A S}} \bmod n_{A S}
\end{array}\right. \\
\left\{\begin{array}{l}
A S \times a_{2}=s_{2}^{e_{A S}} \bmod n_{A S} \Rightarrow \\
A S \times a_{2}^{\prime}=s_{2}^{\prime e_{A S}} \bmod n_{A S}
\end{array}\right. \\
\left\{\begin{array}{l}
A S \times y_{1}=s_{3}^{e_{A S}} \bmod n_{A S} \Rightarrow \\
A S \times y_{1}^{\prime}=s_{3}^{\prime e_{A S}} \bmod n_{A S}
\end{array}\right. \\
\left\{\begin{array}{l}
A S \times y_{2}=s_{4}^{e_{A S}} \bmod n_{A S} \Rightarrow \\
A S \times y_{2}^{\prime}=s_{4}^{\prime e_{A S}} \bmod n_{A S} .
\end{array}\right.
\end{gathered}
$$


This attack can be employed on Asaar et al. (2008a) in a similar way. Furthermore by using one generator $g$ instead of two generator $g$ and $h$, this attack can be employed on Lin et al. (2003), Asaar et al. (2008b) and Hwang et al. (2005) in a similar way. However since in Rodríguez-Henríquez et al. (2007) the computations are done in different interconnected modules, while computation of multiplications, we can not forge new valid ticket similar to this scheme.

Second attack. A malicious voter can exchange the values of $a_{1}, s_{1}$ with $y_{1}, s_{3}$ respectively and compute new values for $s_{5}$ as $s_{5}=r^{-1} \cdot\left(m y_{1}-\left(k_{1}+k_{2}\right)\right) \bmod$ $(p-1)$. By these changes he will be able to produce new forged ticket which passes the validations of the protocol.

In a similar way by exchanging the values of $a_{2}, s_{2}$ with $y_{2}, s_{4}$ respectively and computing new value for $s_{6}$ as $s_{6}=r^{-1}$. $\left(m y_{2}-\left(2 k_{1}+k_{2}\right)\right) \bmod (p-1)$ he can produce another forged ticket.

Also by simultaneously applying the mentioned changes he can produce another forged ticket.

Moreover since the exchanging the sequence of the parameters in a valid ticket, is not checked in Asaar et al. (2008a), Lin et al. (2003), Asaar et al. (2008b), Hwang et al. (2005), and Rodríguez-Henríquez et al. (2007). This attack can be employed on them in a similar way.

\subsubsection{The attack on perceptibility of double voter}

In the scheme, it is supposed that the voter is trusted to choose exponents in the form described in equation (1). However, a malicious voter can deviate from this form, choose other value for $k_{1}$ such as $k_{1}^{\prime}$ and compute the value of $w_{2}^{\prime}=h^{k_{1}^{\prime}} b_{2}^{e_{A S}}$ and fulfil the process of acquiring a valid ticket. In this situation, if a malicious voter votes more than once, TCS will not be able to determine the identity of him.

In Hwang scheme since the voter is trusted to choose exponent on the generators $g$ and $h$ in his share of the keys in the prescribed manner, this attack is applicable on it. The similar situations are held in Asaar et al. (2008a). However since the schemes (Lin et al., 2003; Asaar et al., 2008b; Hwang et al., 2005; Rodríguez-Henríquez et al., 2007) use only one generator $g$ instead of two generators $g$ and $h$, and consequently there is no assumption on the exponents of the generators which the voters must be trusted to obey them. So the attack is not applicable on them.

\section{The new electronic voting scheme}

In the new scheme in order to get vulnerability to the first and second attacks on unforgeability of tickets, multiplicative structure of the keys in the signatures is replaced by additive structure and the generators $g$ and $h$ are chosen in a way that the discrete logarithm of them be unknown. Furthermore, in previous schemes the malicious voter can choose exponents in such way that no authority could trace him if double voting occurs. In order to protect the scheme against the third attack on unforgeability of tickets, we allowed the voter to choose exponent as he desire and by introducing new key structure, the perceptibility of double voting is satisfied. 
In addition, in the new scheme by effectively blinding the signatures of $A S$ and increasing the number of unknown parameters, our new scheme resists the attacks on anonymity of voter.

\subsection{Our proposed scheme}

Our proposed scheme also has composed of three phases of the former scheme. Before starting the protocol, voters must get their certificate from certificate authority. Let $\mathbb{Z}_{p}^{*}$ be a multiplicative group such that solving discrete logarithm problem in it is hard. Furthermore suppose that $q$ be a large prime number such that $q \mid p-1$. In our scheme the certificate authority chooses a generator $g$ of order $q$ for multiplicative group $\mathbb{Z}_{p}^{*}$ and stores it beside the other information in voter's certificate.

\subsubsection{Voting and ticket obtaining phase}

Before starting this phase certificate authority chooses a generator $h$ of order $q$ in $\mathbb{Z}_{p}^{*}$ for AS and publishes it. Then the following steps are done:

a A voter $V$ chooses three blinding factors $b_{1}, b_{2}$ and $b_{3}$ as well as two random numbers $r_{1}$ and $r_{2}$ such that $r_{1}, r_{2}<q / 2$. Then, $V$ computes $w_{1}, w_{2}$ and $w_{3}$ by the following equations:

$$
\begin{aligned}
& w_{1}=\left(g^{r_{1}}+h^{r_{2}}\right) \cdot b_{1}^{e_{A S}} \bmod n_{A S} \\
& w_{2}=g^{r_{1}} b_{2}^{e_{A S}} \bmod n_{A S} \\
& w_{3}=h^{r_{2}} b_{3}^{e_{A S}} \bmod n_{A S} .
\end{aligned}
$$

After that, the voter sends $\left\{V, A S, \operatorname{Cert}_{V}, t, w_{1}, w_{2}, w_{3}\right.$, $\left.\left(\left(w_{1}\left\|w_{2}\right\| w_{3} \| t\right)^{d_{V}}\right) \bmod n_{V}\right\}$ to $A S$.

b $A S$ first verifies the validity of the timestamp $t$ and the certificate $C e r t_{V}$ and then by using the certificate it verify the signature $\left\{\left(\left(w_{1}\left\|w_{2}\right\| w_{3} \| t\right)^{d_{V}}\right) \bmod n_{V}\right\}$. If all verifications are successful, $A S$ chooses $k_{i}, k_{j} \in \mathbb{Z}_{q}^{*}$ such that $k_{i}, k_{j}<q / 2$ randomly and computes $k_{2}=k_{i}+k_{j}$ in a way that $k_{2}$ is a fresh value and has not been already used and computes:

$$
\begin{aligned}
w_{4} & =\left(k_{i}\left\|k_{j}\right\| t\right)^{e_{v}} \bmod n_{v} \\
w_{5} & =\left(A S \times\left(w_{1}\right)\right)^{d_{A S}} \bmod n_{A S} \\
& =\left(A S \times\left(a_{1}+a_{2}\right)\right)^{d_{A S}} \times b_{1} \bmod A S \\
w_{6} & =\left(A S \times\left(w_{2} w_{3}+w_{2} w_{3} \cdot g^{k_{i}} h^{k_{j}}\right)\right)^{d_{A S}} \bmod n_{A S} \\
& =\left(A S \times\left(a_{1} a_{2}+y_{1} y_{2}\right)\right)^{d_{A S}} \cdot b_{2} b_{3}, \bmod _{A S} .
\end{aligned}
$$

where $a_{1}=g^{r_{1}}, a_{2}=h^{r_{2}}, y_{1}=g^{r_{1}+k_{i}}$, and $y_{2}=h^{r_{2}+k_{j}}$. Then, $A S$ delivers the messages $\left\{A S, V, w_{4}, t,\left(w_{5}\left\|w_{6}\right\| t\right)^{e_{V}} \bmod n_{V}\right\}$ to $V$. Note that $A S$ also records $k_{2}$ along with $V$ 's identity in its database. 
c $\quad V$ decrypts $w_{4}$ to obtain $k_{i}$ and $k_{j}$. Thus, $V$ can calculate $y_{1}$ and $y_{2}$ by multiplying $a_{1}$ and $a_{2}$ with $g^{k_{i}}$ and $h^{k_{j}}$ respectively. In addition, $V$ also computes the signatures $s_{1}, s_{2}$, and $s_{3}$ by the following equations:

$$
\begin{aligned}
s_{1} & =w_{5} \times b_{1}^{-1} \bmod n_{A S} \\
& =\left(A S \times\left(a_{1}+a_{2}\right)\right)^{d_{A S}} \bmod n_{A S} \\
s_{2} & =w_{6} \times b_{2}^{-1} b_{3}^{-1} \bmod n_{A S} \\
& =\left(A S \times\left(a_{1} a_{2}+y_{1} y_{2}\right)\right)^{d_{A S}} \bmod n_{A S} .
\end{aligned}
$$

d $\quad V$ applies the ElGamal digital signature scheme to sign the voting content $m$. Let $y_{1}$ and $y_{2}$ be the public keys of the ElGamal Cryptosystem, and $x_{1}=r_{1}+k_{i}$ and $x_{2}=r_{2}+k_{j}$ be the corresponding private keys, such that $y_{1}=g^{x_{1}} \bmod p$ and $y_{2}=h^{x_{2}} \bmod p . V$ generates two signatures $\left(a_{1}, s_{3}\right)$ and $\left(a_{1}, s_{4}\right)$ of the voting content $m$ by using the following equations:

$$
\begin{aligned}
& s_{3}=x_{1}^{-1}\left(m a_{1}-r_{1}\right) \bmod q \\
& s_{4}=x_{2}^{-1}\left(m a_{2}-r_{2}\right) \bmod q .
\end{aligned}
$$

Then the voting ticket can be computed as

$$
T=\left\{s_{1}\left\|s_{2}\right\| s_{3}\left\|s_{4}\right\| a_{1}\left\|a_{2}\right\| y_{1}\left\|y_{2}\right\| m\right\} .
$$

Note that due to condition imposed on $r_{1}, r_{2}, k_{i}$ and $k_{j}$, we are sure that $x_{1}^{-1}$ and $x_{2}^{-1}$ exist.

\subsubsection{The voting and tickets collecting phase}

a $\quad V$ sends the voting ticket $T$ to $V S$.

b $\quad V S$ verifies the validity of $a_{1}, a_{2}, y_{1}$ and $y_{2}$ by checking the following equations:

$$
\begin{aligned}
& A S \times\left(a_{1}+a_{2}\right) \stackrel{?}{=} s_{1}^{e_{A S}} \bmod n_{A S} \\
& A S \times\left(a_{1} a_{2}+y_{1} y_{2}\right) \stackrel{?}{=} s_{2}^{e_{A S}} \bmod n_{A S} .
\end{aligned}
$$

If the above equations hold, $V S$ further verifies the signatures $\left(a_{1}, y_{1}, s_{3}\right)$ and $\left(a_{2}, y_{2}, s_{4}\right)$ of the voting content $m$ by checking the following equations:

$$
\begin{aligned}
& y_{1}^{s_{3}} a_{1} \stackrel{?}{=} g^{m a_{1}} \bmod p \\
& y_{2}^{s_{4}} a_{2} \stackrel{?}{=} h^{m a_{2}} \bmod p .
\end{aligned}
$$

If both verifications succeed, $V S$ stores $T$ in its database.

c After the voting time expired, VS sends all the collected tickets to TCS. 


\subsubsection{The tickets counting phase}

Upon receiving all tickets from the $V S, T C S$ first verifies if there are double voting tickets by checking if the values of $a_{1}, a_{2}, y_{1}, y_{2}$ of one ticket has not occurred in another ticket in one of the following form:

$$
\begin{aligned}
T^{\prime}= & \left\{s_{1}^{\prime}\left\|s_{2}^{\prime}\right\| s_{3}^{\prime}\left\|s_{4}^{\prime}\right\| a_{1}\left\|a_{2}\right\| y_{1}\left\|y_{2}\right\| m^{\prime}\right\} \\
& \left\{s_{1}^{\prime}\left\|s_{2}^{\prime}\right\| s_{3}^{\prime}\left\|s_{4}^{\prime}\right\| y_{1}\left\|a_{2}\right\| a_{1}\left\|y_{2}\right\| m^{\prime}\right\} \\
& \left\{s_{1}^{\prime}\left\|s_{2}^{\prime}\right\| s_{3}^{\prime}\left\|s_{4}^{\prime}\right\| a_{1}\left\|y_{2}\right\| y_{1}\left\|a_{2}\right\| m^{\prime}\right\} \\
& \left\{s_{1}^{\prime}\left\|s_{2}^{\prime}\right\| s_{3}^{\prime}\left\|s_{4}^{\prime}\right\| y_{1}\left\|y_{2}\right\| a_{1}\left\|a_{2}\right\| m^{\prime}\right\}
\end{aligned}
$$

if one of these forms occurs in another ticket, double voting occurs and TCS can compute the value of $k_{2}$ corresponding to the malicious voter as follows:

$$
\begin{aligned}
x_{1} & =\frac{m^{\prime} a_{1}-m a_{1}}{s_{3}^{\prime}-s_{3}} \bmod q \\
x_{2} & =\frac{m^{\prime} a_{2}-m a_{2}}{s_{4}^{\prime}-s_{4}} \bmod q \\
r_{1} & =m a_{1}-s_{3} x_{1} \bmod q \\
r_{2} & =m a_{2}-s_{4} x_{2} \bmod q \\
k_{i} & =\left|x_{1}-r_{1}\right| \bmod q \\
k_{j} & =\left|x_{2}-r_{2}\right| \bmod q \\
k_{2} & =k_{i}+k_{j} \bmod q
\end{aligned}
$$

in which $|X|$ means that:

$$
|X|= \begin{cases}X \bmod q & \text { if } X<q / 2 \\ q-X \bmod q & \text { otherwise. }\end{cases}
$$

Then, $T C S$ can identify the malicious voter by cooperating with $A S$ and searching $A S$ 's database to find out which voter is associated with the unique random number $k_{2}$.

\subsection{Analysis of the new scheme}

\subsubsection{Security analysis of the scheme}

The problem of previous works is their multiplicative structure that causes them vulnerable to multiplicative attack which is presented in this paper. In our scheme by replacing multiplicative structure of the keys in the signatures by additive structure and choosing $g$ and $h$ by certificate authority, in a way that no one knows the discrete logarithm of them, we could make our scheme resistant against the first attack on unforgeability of tickets. Since $k_{i}$ and $k_{j}$ must be less than $q / 2$ for all voters, by the help introducing the operation $|X|$, we can obtain the identity of malicious voters who employ the second attack on unforgeability of tickets. On the other hand in previous schemes the voter was trusted to choose exponent in his 
share of the keys in the prescribed manner. However malicious voter can choose exponents in such way that no authority can trace him if double voting occurs. In order to preclude this attack we allowed the voter to choose exponent as he desires and by introducing new key structure the perceptibility of double voting claimed in previous schemes satisfied. The other essential future of the e-voting is protecting anonymity of voters. In this paper it is showed that anonymity of voter can easily be removed without double voting. However in the new scheme by effectively blinding the signatures of $A S$ and increasing the number of unknown parameters, the scheme is resistant against the proposed attacks.

\subsubsection{Efficiency of the new scheme}

By comparing number of multiplications and number of exponentiations we found that our scheme is more efficient than Hwang scheme. Table 2 expresses the comparison between our scheme and Hwang scheme.

Table 2 Comparing efficiency of Hwang scheme and our scheme

\begin{tabular}{lccccccc}
\hline & \multicolumn{3}{c}{ Multiplication } & & \multicolumn{3}{c}{ Exponentiation } \\
\cline { 2 - 4 } Schemes & Phase 1 & Phase 2 & Phase 3n & & Phase 1 & Phase 2 & Phase 3 \\
\hline Hwang et al. & 18 & 8 & 6 & & 22 & 8 & 0 \\
Our scheme & 16 & 8 & 10 & & 18 & 6 & 0 \\
\hline
\end{tabular}

\section{Conclusion}

In this paper we discussed the weaknesses of Hwang scheme in satisfying the properties of unforgeability of the tickets and perceptibility of double voter and anonymity of voters. We mentioned the applicability of these attacks on other scheme in the generation of $\mathrm{Mu}$-Varadharajan protocols. Furthermore we proposed a new scheme and showed that this scheme beside the resistance against the attack which have been proposed until now, satisfy the anonymity and unforgeability of the ticket.

\section{Acknowledgement}

The authors would like to thank the Research Deputy Chancellor of Sharif University of Technology for financially support of this research.

\section{References}

Asaar, M., Mohajeri, J. and Salmasizadeh, M. (2008a) 'Security modification for the Hwang-Wen-Hwang's e-voting scheme', Proceedings of International Conference on Security and Management (SAM'08), Vol. 139, pp.486-490.

Asaar, M., Mohajeri, J. and Salmasizadeh, M. (2008b) 'Another security improvment over the Lin et al.'s electronic voting scheme', International Journal of Electronic Security and Digital Forensics, Vol. 1, No. 4, pp.413-422. 
Benaloh, J. (1987) Verifiable Secret-Ballot Elections, PhD Thesis, Yale University, Department of Computer Science, New Haven, CT, September.

Chaum, D. (2004) 'Secret-ballot receipts: true voter-verifiable elections', IEEE Security and Privacy Magazine, Vol. 2, January-February, pp.38-47.

Chaum, D., Rayan, P. and Schneider, S. (2005) 'A practical, voter-verifiable election scheme', Proc. 10th European Symposium on Research in Computer Security (ESORICS'05), Vol. 3679, LNCS, Springer, pp.118-139.

Chien, H., Jan, J. and Tseng, Y. (2003) 'Cryptanalysis on Mu-Varadharajan's e-voting schemes', Appl. Math. Comput., Vol. 139, Nos. 2-3, pp.525-530.

Chum, D. (1988) 'Elections with unconditionally-secret ballots and disruption equivalent to breaking RSA', Advances in Cryptology-Eurocrypt'88, Vol. 330, LNCS, Springer, pp.177-182.

ELGamal, T. (1985) 'A public-key cryptosystem and a signature scheme based on discrete logarithm', IEEE Transactions on Information Theory, Vol. 31, July, pp.469-472.

Fujioka, A., Okamoto, T. and Ohta, K. (1988) 'A practical secret voting scheme for large scale elections', Advances in Cryptology-AUSCRYPT'92, Vol. 718, LNCS, Springer, pp.244-251.

Hirt, M. and Sako, K. (2000) 'Efficient receipt-free voting based on homomorphic encryption', Advances in Cryptography-Eurocrypt 2000, Vol. 1807, LNCS, Springer, pp.539-556.

Hwang, S., Wen, H. and Hwang, T. (2005) 'On the security enhancement for anonymous secure e-voting over computer network', Computer Standards and Interfaces, Vol. 27, No. 2, pp.163-168.

Juang, W. and Lei, C. (1997) 'A secure and practical electronic voting scheme for real world environments', IEICE Transaction on Fundamentals of Electronics, Communications and Computer Science, Vol. E80, No. 1, January, pp.64-71.

Lee, B., Boyd, C., Dawson, E., Kim, K., Yang, J. and Yoo, S. (2004) Providing receipt-freeness in mixnet-based voting protocols', Proceedings of Information Security and Cryptology (ICISC'03), Vol. 2971, LNCS, Springer, pp.245-258.

Lin, I., Hwang, M. and Chang, C. (2003) 'Security enhancement for anonymous secure e-voting over a network', Computer Standards and Interfaces, Vol. 25, pp.131-139.

Mu, Y. and Varadharajan, V. (1998) 'Anonymous secure e-voting over a network', Proceedings of the 14th Annual Computer Security Application Conference. IEEE Computer Society, Washington DC, USA, pp.293-299.

Rodríguez-Henríquez, F., Ortiz-Arroyo, D. and García-Zamora, C. (2007) 'Yet another improvement over the Mu-Varadharajan e-voting protocol', Computer Standards and Interfaces, Vol. 29, pp.471-480.

Yang, C., Lin, C. and Yang, H. (2004) 'Improved anonymous secure e-voting over a network', Informatim \& Security, Vol. 15, No. 2, pp.185-191. 